\title{
Effect of using amino acids in the freeze-drying of siRNA lipoplexes on gene knockdown in cells after reverse transfection
}

\author{
MIN TANG and YOSHIYUKI HATTORI \\ Department of Molecular Pharmaceutics, Hoshi University, Tokyo 142-8501, Japan
}

Received March 23, 2021; Accepted June 25, 2021

DOI: $10.3892 /$ br.2021.1448

\begin{abstract}
Recently, small interfering RNA (siRNA)/cationic liposome complexes (siRNA lipoplexes) have become a crucial research tool for studying gene function. Easy and reliable siRNA transfection with a large set of siRNAs is required for the successful screening of gene function. Reverse (Rev)-transfection with freeze-dried siRNA lipoplexes is validated for siRNA transfection with a large set of siRNAs in a multi-well plate. In our previous study, it was shown that Rev-transfection with siRNA lipoplexes freeze-dried in disaccharides or trisaccharides resulted in long-term stability with a high level of gene-knockdown activity. In the present study, the effects of amino acids used as cryoprotectants in the freeze-drying of siRNA lipoplexes on gene knockdown via Rev-transfection were assessed. A total of 15 types of amino acids were used to prepare freeze-dried siRNA lipoplexes, and it was found that the freeze-drying of siRNA lipoplexes with amino acid concentrations $>100 \mathrm{mM}$ strongly suppressed targeted gene expression regardless of the amino acid type; however, some amino acids strongly upregulated or downregulated gene expression in the cells transfected with negative control siRNA. Amongst the amino acids tested, the presence of asparagine showed specific gene-knockdown activity, forming large cakes after freeze-drying and retaining a favorable siRNA lipoplex size after rehydration. These findings provide valuable information regarding amino acids as cryoprotectants for Rev-transfection using freeze-dried siRNA lipoplexes for the efficient delivery of siRNA into cells.
\end{abstract}

\section{Introduction}

Small interfering RNAs (siRNAs) inhibit the expression of mRNAs bearing complementary sequences (1). The first siRNA therapeutic, patisiran, was approved by the FDA in 2018,

Correspondence to: Professor Yoshiyuki Hattori, Department of Molecular Pharmaceutics, Hoshi University, 2-4-41 Ebara, Shinagawa-ku, Tokyo 142-8501, Japan

E-mail: yhattori@hoshi.ac.jp

Key words: cationic liposome, small interfering RNA, reverse transfection, amino acids, freeze-drying and the second siRNA therapeutic, givosiran, was approved the following year (2). Several studies have focused on RNA therapy, especially siRNA-based therapeutics. Screening with siRNA libraries is an efficient method for identifying genes as therapeutic targets (3). For screening with a large set of siRNAs, reliable and reproducible transfections in multi-well plates is required (4). However, siRNAs are unable to diffuse passively into cells on their own; therefore, they are often introduced into cells using cationic liposomes (5). Several studies have demonstrated that siRNA and cationic liposome complexes (siRNA lipoplexes) are unstable when stored in solution at room temperature (6). A relatively simple method to store siRNA lipoplexes at room temperature for longer periods of time is to transform the siRNA lipoplex suspension into a freeze-dried siRNA lipoplex. Solid-phase reverse (Rev)-transfection using freeze-dried siRNA lipoplexes is a valid means of easy and reliable siRNA transfection with cationic liposomes $(7,8)$. In Rev-transfection with freeze-dried siRNA lipoplexes, siRNA lipoplex solutions are freeze-dried in multi-well plates, and at the time of transfection, cell suspensions are added to the wells.

Freeze-drying is widely used to stabilize liposomes for long-term storage at room temperature $(9,10)$. However, siRNA lipoplexes increase in size following dehydration-rehydration cycles, resulting in a decrease in gene knockdown activity if they are freeze-dried without appropriate stabilizers (7). Although cryoprotectants, including saccharides (such as sucrose and trehalose) (6,11-13) and amino acids (14), are employed to stabilize liposomes and lipid nanoparticles during the freeze-drying and rehydration processes, the mechanism of protection of these molecules is not yet fully understood. After decades of studying saccharides, two hypotheses, water replacement and vitrification, have been well accepted. In the water replacement hypothesis, instead of water molecules, saccharides associate with the polar head groups of the hydrated phospholipids of the liposomal membrane, stabilizing the liposome (15). Alternatively, the vitrification hypothesis indicates that saccharides around the bilayer form a vitreous layer, which depresses the transition temperature of the lipids (16). Alternatively, amino acids are not only able to offer hydrogen bonds, but also provide electrostatic interactions for effective lyophilization (14). To improve the stability of proteins, amino acids are often added as stabilizing excipients to prevent protein inactivation during freeze-drying and storage $(17,18)$. Furthermore, the addition of lysine to liposomes during 
freeze-drying appears to have protective effects similar to those of trehalose (14). However, to the best of our knowledge, there are no studies assessing the application of amino acids as cryoprotectants in the freeze-drying of siRNA lipoplexes.

In our previous study, it was shown that Rev-transfection with siRNA lipoplexes freeze-dried in the presence of a disaccharide or trisaccharide solution resulted in a high level of gene-knockdown activity without exerting a notable cytotoxic effect $(7,8)$. In the present study, to investigate the appropriate use of amino acids as cryoprotectants to retain this high efficiency of Rev-transfection using siRNA lipoplexes, freeze-dried siRNA lipoplexes were prepared with 15 types of amino acids, and their efficiency in gene knockdown in cells was assessed. The presence of amino acids during the freeze-drying of siRNA lipoplexes resulted in gene knockdown activity; however, certain amino acids strongly upregulated or downregulated gene expression in cells transfected with negative control siRNA. In addition, the types of amino acids used during freeze-drying affected the size of the siRNA lipoplexes following rehydration. Amongst the amino acids tested in the present study, siRNA lipoplexes freeze-dried with asparagine showed specific gene suppression, forming large cakes after freeze-drying, whilst retaining a relatively small size following rehydration. The results of the present study provide valuable information regarding the optimal amino acids for use as cryoprotectants for Rev-transfection using freeze-dried siRNA lipoplexes.

\section{Materials and methods}

Materials. 1,2-Dioleoyl-3-trimethylammonium-propane methyl sulfate salt (DOTAP) was obtained from Avanti Polar Lipids Inc. 1,2-Dioleoyl-sn-glycero-3-phosphoethanolamine (DOPE, COATSOME; cat. no. ME-8181) was obtained from NOF Co., Ltd. L-Alanine, L-arginine hydrochloride, L-asparagine monohydrate, L-cysteine, L-glutamine, glycine, L-histidine, L-isoleucine, L-leucine, L-lysine, L-methionine, L-proline, L-serine, L-threonine and L-valine were obtained from Wako Pure Chemical Industries, Ltd. All the other chemicals used were of the highest available grade, and were obtained from commercial sources.

Small interfering (si)RNAs. siRNAs targeting nucleotides of firefly luciferase (Luc siRNA) and non-targeting siRNA control (Cont siRNA) as a negative control for Luc siRNA were synthesized by Sigma Genosys. The siRNA sequences of the Luc siRNA were as follows: Sense strand, 5'-CCGUGGUGUUCGUGUCUAAGA-3' and antisense strand, 5'-UUAGACACGAACACCACGGUA-3' (19), and the siRNA sequences of the Cont siRNA were as follows: Sense strand, 5'-GUACCGCACGUCAUUCGUAUC-3' and antisense strand, 5'-UACGAAUGACGUGCGGUACGU-3' (20).

Appearance of cakes after freeze-drying the amino acid solution. To compare the cake volumes after freeze-drying, $5 \mathrm{ml}$ $100 \mathrm{mM}$ alanine, arginine, asparagine, cysteine, glutamine, glycine, histidine, isoleucine, leucine, lysine, methionine, proline, serine, threonine and valine solution were transferred into a $5 \mathrm{ml}$ vial, and then frozen at $-80^{\circ} \mathrm{C}$, followed by drying under a high vacuum pressure (10-20 Pa) using a FDU-540 freeze-dryer (Tokyo Rikakikai Co.) equipped with a DRC-2L dry chamber (Tokyo Rikakikai Co.).

Preparation of cationic liposomes and siRNA lipoplexes. Cationic liposomes were prepared from DOTAP and DOPE at a molar ratio of 1:1 (21). For the preparation of cationic liposomes using the thin-film hydration method, DOTAP and DOPE were dissolved in chloroform, and the chloroform was evaporated under vacuum on a rotary evaporator at $60^{\circ} \mathrm{C}$ to obtain a thin film. The thin film was hydrated with water at $60^{\circ} \mathrm{C}$ through vortexing. The hydrated liposomes were placed in an eggplant flask and sonicated using a bath-type sonicator (Bransonic $^{\circledR} 2510 \mathrm{~J}-\mathrm{MTH}, 42 \mathrm{kHz}, 100 \mathrm{~W}$; Branson UL Trasonics Co.) for 5-10 min at room temperature.

To prepare cationic liposome/siRNA complexes (siRNA lipoplexes), each liposome preparation was added to siRNA at a charge ratio (+:-) of 4:1 via vortex mixing for $10 \mathrm{sec}$, leaving the solution at room temperature for $15 \mathrm{~min}$. The charge ratio (+:-) of liposomes:siRNA is expressed as the molar ratio of cationic lipid to siRNA phosphate.

Size of reconstituted siRNA lipoplexes. To measure the size of siRNA lipoplexes, siRNA lipoplexes were formed through the addition of cationic liposomes to $5 \mu \mathrm{g}$ Cont siRNA with vortex-mixing for $10 \mathrm{sec}$ and leaving the solution at room temperature for $15 \mathrm{~min}$. In the preparation of freeze-dried siRNA lipoplexes, each lipoplex containing $5 \mu \mathrm{g}$ Cont siRNA was diluted in $933 \mu 1100 \mathrm{mM}$ amino acid solution sterilized using a $0.45-\mu \mathrm{m}$ filter [125 $\mu \mathrm{l}$ amino acid solution per $50 \mathrm{pmol}(0.67 \mu \mathrm{g})$ siRNA], and then transferred to a 6-well plate, followed by freezing at $-80^{\circ} \mathrm{C}$. The frozen plates were dried under a high vacuum using a freeze-dryer. Freeze-dried siRNA lipoplexes were reconstituted to an appropriate volume ( $\sim 1 \mathrm{ml})$ with water, and the particle size (cumulant average particle size) and polydispersity index (PDI) of the siRNA lipoplexes were measured using the cumulant method with a ELS-Z2 light-scattering photometer (Otsuka Electronics Co., Ltd.) at $25^{\circ} \mathrm{C}$.

Cell culture. Human breast cancer MCF-7 cells stably expressing firefly luciferase (MCF-7-Luc), constructed via transfection with a pcDNA3 plasmid containing the firefly luciferase gene from the plasmid psiCHECK2 (Promega Corporation), were donated by Dr Kenji Yamato of the University of Tsukuba. MCF-7-Luc cells were grown in RPMI-1640 medium (Wako Pure Chemical Industries, Ltd.) supplemented with $10 \%$ heat-inactivated FBS (Invitrogen; Thermo Fisher Scientific, Inc.) and $1.2 \mathrm{mg} / \mathrm{ml}$ G418 (Santa Cruz Biotechnology, Inc.) at $37^{\circ} \mathrm{C}$ in a $5 \% \mathrm{CO}_{2}$ humidified atmosphere.

Effect of amino acid types used in the freeze-drying of siRNA lipoplexes on gene knockdown by Rev-transfection. For Rev-transfection, siRNA lipoplexes were formed through the addition of cationic liposomes to $50 \mathrm{pmol}$ Cont siRNA or Luc siRNA by vortexing for $10 \mathrm{sec}$, and then leaving the solutions to stand at room temperature for $15 \mathrm{~min}$. Each lipoplex was diluted in a $125 \mu \mathrm{l}$ solution containing different concentrations of amino acids $(10,25,50,100$ or $150 \mathrm{mM})$, transferred to a 12 -well plate ( $50 \mathrm{pmol}$ siRNA/well), followed by freezing at $-80^{\circ} \mathrm{C}$. The frozen plates were dried under high vacuum using 
a freeze-dryer and stored at room temperature in a desiccator until required. For Rev-transfection using freeze-dried siRNA lipoplexes, MCF-7-Luc cells $\left(1 \times 10^{5}\right.$ cells) were suspended in $1 \mathrm{ml}$ culture medium supplemented with $10 \% \mathrm{FBS}$, and this suspension was added to each well (final siRNA concentration, $50 \mathrm{nM}$ ). The medium, after the rehydration of siRNA lipoplexes freeze-dried with $10,25,50,100$ or $150 \mathrm{mM}$ amino acid solutions, contained $1.25,3.125,6.25,12.5$ or $18.75 \mathrm{mM}$ extra amino acids, respectively. A total of $48 \mathrm{~h}$ after transfection, the cells were lysed through the addition of $125 \mu \mathrm{l}$ cell lysis buffer (Pierce Luciferase Cell Lysis Buffer; Thermo Fisher Scientific Inc.) and subjected to one cycle of freezing $\left(-80^{\circ} \mathrm{C}\right)$ and thawing $\left(37^{\circ} \mathrm{C}\right)$, followed by centrifugation at $15,000 \mathrm{x} \mathrm{g}$ for $10 \mathrm{sec}$ at $4^{\circ} \mathrm{C}$. A total of $10 \mu \mathrm{l}$ aliquots of the cell lysate supernatants were mixed with $50 \mu 1$ PicaGene MelioraStar-LT Luminescence Reagent (Toyo Ink Mfg. Co., Ltd.), and the luminescence was measured as counts per second (cps) with a chemoluminometer (ARVO X2; Perkin Elmer, Inc.). The protein concentration of the supernatants was determined using BCA reagent (Pierce BCA Protein assay kit; Thermo Fisher Scientific Inc.), with bovine serum albumin (BSA; Sigma-Aldrich; Merck KGaA) as a standard, and the luciferase activity (cps/ $\mu \mathrm{g}$ protein) was calculated. Luciferase activity (\%) was calculated relative to the luciferase activity (cps/ $\mu$ g protein) of untransfected cells.

To investigate the effect of amino acids on luciferase expression in MCF-7-Luc cells, $125 \mu 1100 \mathrm{mM}$ amino acid solution was transferred to a 12-well plate, followed by freeze-drying. MCF-7-Luc cells $\left(1 \times 10^{5}\right)$ were suspended in $1 \mathrm{ml}$ medium supplemented with $10 \%$ FBS and the resulting cell suspension was added to the wells. Luciferase activity was measured as described above.

Cytotoxicity caused by Rev-transfection with freeze-dried siRNA lipoplexes. Each lipoplex containing 5 pmol Cont siRNA was diluted with a $12.5 \mu \mathrm{l}$ solution containing $100 \mathrm{mM}$ amino acid, and then transferred to each well of a 96-well plate (5 pmol siRNA/well). After freezing at $-80^{\circ} \mathrm{C}$, the plates were dried under a high vacuum using a freeze-dryer. MCF-7-Luc cells $\left(1 \times 10^{4}\right)$ were suspended in $100 \mu 1$ culture medium supplemented with $10 \% \mathrm{FBS}$, and the suspension was added to each well (final siRNA concentration of $50 \mathrm{nM}$ ). After $24 \mathrm{~h}$ of incubation, cell viability was determined using a Cell Counting Kit-8 assay (Dojindo Laboratories). The relative cell viability (\%) was calculated as the percentage of the cells added to the wells without freeze-dried lipoplexes.

Statistical analysis. Data are presented as the mean \pm standard deviation of at least three repeats. The statistical significance of the differences between mean values was determined using Student's t-test or a one-way ANOVA followed by a post-hoc Tukey's test in GraphPad Prism version 4.0 (GraphPad Software Inc.). $\mathrm{P}<0.05$ was considered to indicate a statistically significant difference.

\section{Results}

Appearance of cakes after freeze-drying amino acid solution. In the present study, 15 amino acids (alanine, arginine, asparagine, cysteine, glutamine, glycine, histidine, isoleucine, leucine, lysine, methionine, proline, serine, threonine and

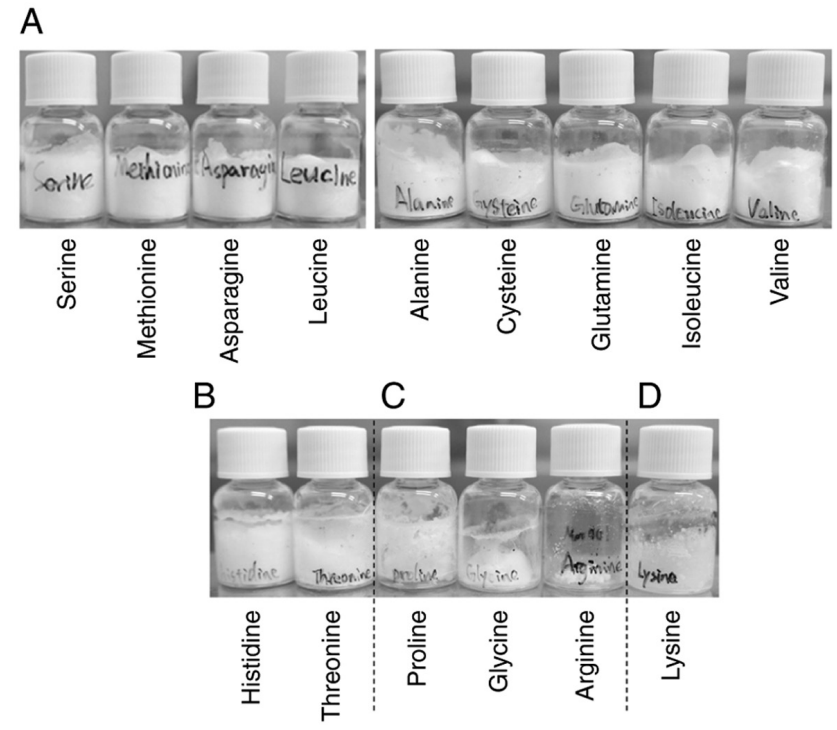

Figure 1. Appearance of cakes after freeze-drying of amino acid solutions. A total of $5 \mathrm{ml} 100 \mathrm{mM}$ alanine, arginine, asparagine, cysteine, glutamine, glycine, histidine, isoleucine, leucine, lysine, methionine, proline, serine, threonine and valine solutions were transferred into $5 \mathrm{ml}$ vials, followed by freezing at $-80^{\circ} \mathrm{C}$. The frozen vials were dried under a high vacuum using a freeze-dryer. (A) Appearance of cakes formed using serine, methionine, asparagine, leucine, alanine, cysteine, glutamine, isoleucine and valine; (B) histidine and threonine; (C) proline, glycine, and arginine; and (D) lysine solutions.

valine) were used as cryoprotectants during the freeze-drying of siRNA lipoplexes, and their effects on gene knockdown efficiency after Rev-transfection using freeze-dried siRNA lipoplexes were investigated. As aspartic acid, glutamic acid, phenylalanine, tyrosine and tryptophan did not completely dissolve in water at a concentration of $100-150 \mathrm{mM}$, these were excluded as potential cryoprotectants. The appearance of cakes (dry powder) after freeze-drying may be an indicator of product quality. Thus, first, a $100 \mathrm{mM}$ amino acid solution was prepared and freeze-dried in a vial. Histidine and threonine formed shrunken cakes (Fig. 1B) after freeze-drying; arginine, glycine and proline formed collapsed cakes (Fig. 1C); and lysine formed puffing cakes (Fig. 1D). In contrast, the other amino acids formed large cakes (Fig. 1A), similar to previously reported disaccharides $(7,8)$. This result suggests that different amino acid types affect the appearance of cakes after freeze-drying.

Effect of amino acid types in freeze-dried siRNA lipoplexes on gene knockdown in Rev-transfected cells. Reverse transfection is a method in which siRNA lipoplexes are attached to the bottom of cell culture plates through freeze-drying, then, at the time of transfection, a cell suspension is added to the culture plate $(7,8)$. To assess the gene-knockdown effects of siRNA lipoplexes freeze-dried in the presence of amino acids, freeze-dried siRNA lipoplexes in 12-well plates were reconstituted with MCF-7-Luc cells suspended in culture medium. Here, DOTAP was used as a cationic lipid and DOPE as a helper lipid, and cationic liposomes were prepared at a molar ratio of 1:1 (8). In our previous study, it was reported that cationic liposomes composed of DOTAP and DOPE could efficiently deliver siRNA into the cells via conventional transfection (forward transfection) and strongly suppress the expression of target genes (>80\% knockdown) (7). siRNA lipoplexes were formed 

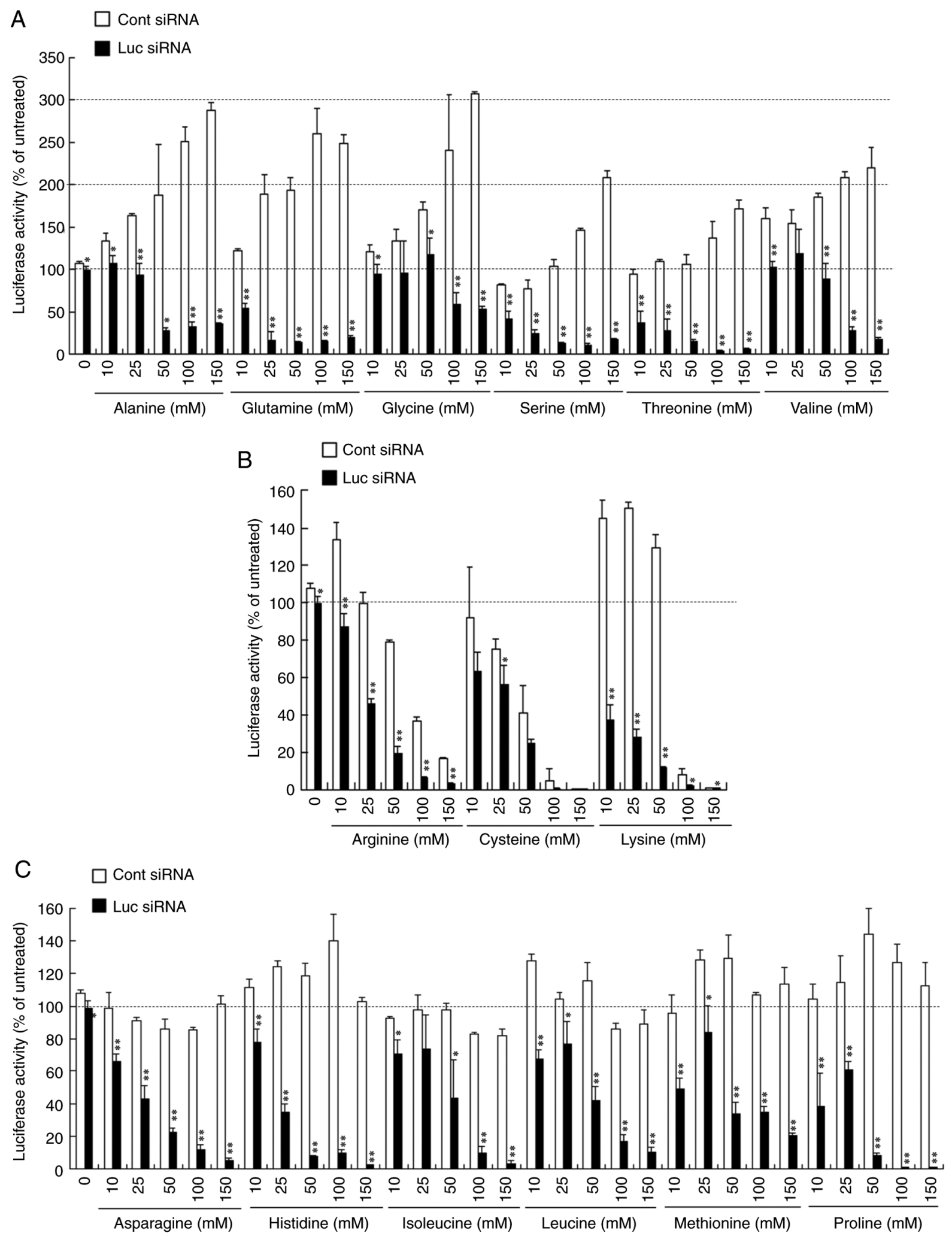

Figure 2. Effect of amino acids in the freeze-drying on the suppression of luciferase expression in MCF-7-Luc cells after reverse transfection with siRNA lipoplexes. Lipoplexes with $50 \mathrm{pmol}$ Cont siRNA or Luc siRNA were diluted in a $125 \mu 1$ solution containing 10, 25, 50, 100 or $150 \mathrm{mM}$ (A) alanine, glutamine, glycine, serine, threonine or valine; (B) arginine, cysteine or lysine; or (C) asparagine, histidine, isoleucine, leucine, methionine or proline, and transferred to 12 -well plates, followed by freeze-drying. MCF-7-Luc cells suspended in culture medium (1 ml) were added to each well, and luciferase assays were performed after incubation at $37^{\circ} \mathrm{C}$ for $48 \mathrm{~h}$. Data are presented as the mean \pm standard deviation of three repeats. ${ }^{*} \mathrm{P}<0.05,{ }^{* *} \mathrm{P}<0.01$ vs. Cont siRNA. Luc, luciferase; Cont, control; siRNA, small interfering RNA; lipoplex, liposome complex.

at a charge ratio (+:-) of 4:1, diluted with solutions containing different concentrations of amino acids, and then added into the wells of a 12-well plate, followed by freeze-drying (Fig. S1).
When siRNA lipoplexes were freeze-dried without amino acids, the lipoplexes with Luc siRNA did not suppress luciferase activity after Rev-transfection (Fig. 2A-C). 


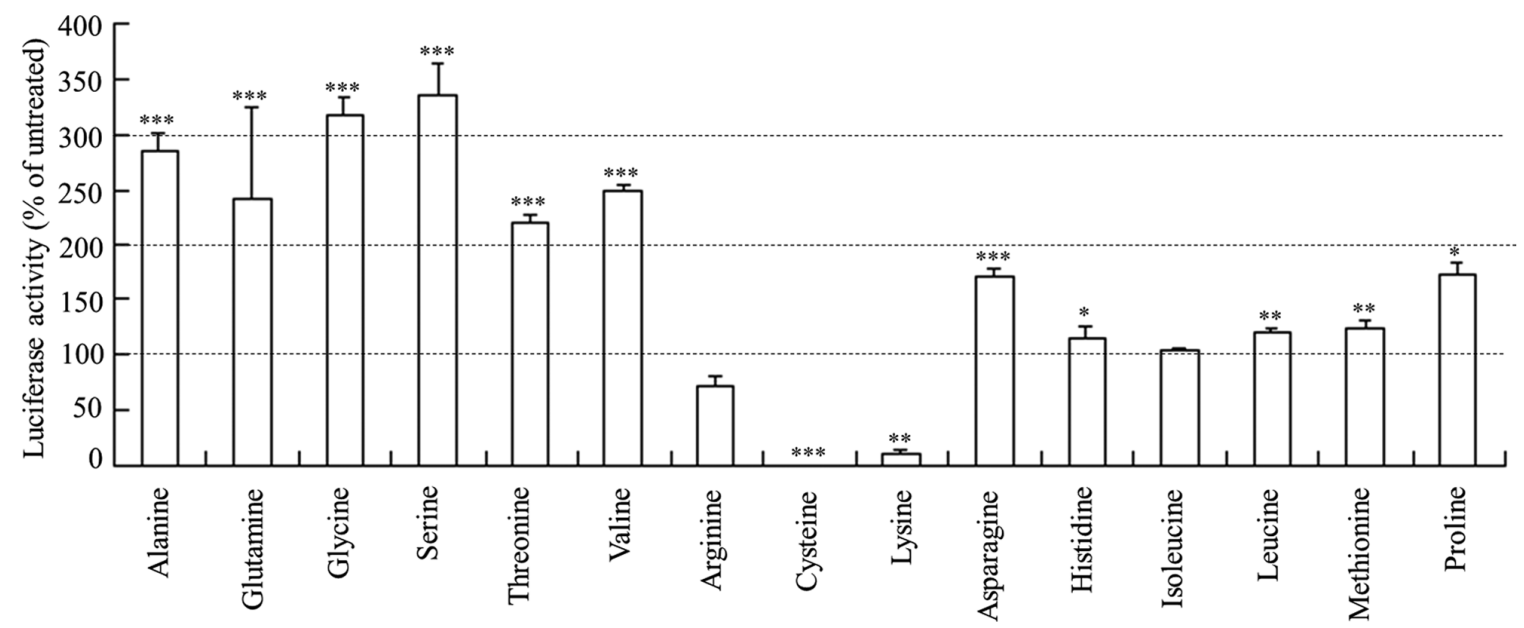

Figure 3. Effect of amino acids on the expression of the luciferase genes in MCF-7-Luc cells. A $125 \mu 1$ solution containing $100 \mathrm{mM}$ amino acids was transferred into each well of a 12-well plate, followed by freeze-drying. MCF-7-Luc cells suspended in culture medium (1 ml) were added to each well, and luciferase assays were performed after incubation at $37^{\circ} \mathrm{C}$ for $48 \mathrm{~h}$. Data are presented as the mean \pm standard deviation of three repeats. ${ }^{*} \mathrm{P}<0.05,{ }^{* * *} \mathrm{P}<0.01$, ${ }^{* * * *} \mathrm{P}<0.001$ vs. untreated cells.

However, increasing concentrations of amino acids used in the freeze-drying of siRNA lipoplexes were associated with increased gene-knockdown activity after Rev-transfection. Freeze-drying of lipoplexes containing Luc siRNA in the presence of 50-100 mM amino acids strongly suppressed luciferase activity regardless of the amino acid type. Interestingly, luciferase activity after Rev-transfection with lipoplexes of Cont siRNA differed depending on the amino acid used. The results could be divided into three groups: The presence of amino acids in freeze-drying that exhibited increased luciferase activity (Fig. 2A), decreased luciferase activity (Fig. 2B) or no notable change in luciferase activity (Fig. 2C). Increasing concentrations of alanine, glutamine, glycine, serine, threonine or valine in the freeze-drying of siRNA lipoplexes increased luciferase activity ( $200 \%$ compared with the untreated cells) after Rev-transfection with lipoplexes of Cont siRNA (Fig. 2A). In contrast, the presence of arginine, cysteine or lysine during freeze-drying decreased luciferase activity at increasing concentrations, whereas at concentrations $>100 \mathrm{mM}$, luciferase activity decreased to $\sim 20 \%$ compared with the untreated cells (Fig. 2B). However, the presence of asparagine, histidine, isoleucine, leucine, methionine or proline during freeze-drying did not notably affect luciferase activity (Fig. 2C). To confirm the effects of amino acids on luciferase activity, MCF-7-Luc cells were added to each well of a multi-well plate, which was freeze-dried with $100 \mathrm{mM}$ amino acids without siRNA lipoplexes (Fig. 3). As a result, the extra amino acids affected luciferase activity similarly to those after Rev-transfection with the lipoplexes of Cont siRNA freeze-dried with amino acids (Fig. 2), indicating that the presence of extra amino acids in the culture medium may affect luciferase expression or luciferase activity.

Cytotoxicity of Rev-transfection with freeze-dried siRNA lipoplexes. Cell viability was measured $24 \mathrm{~h}$ after Rev-transfection with freeze-dried siRNA lipoplexes into MCF-7-Luc cells. The siRNA lipoplexes freeze-dried with arginine, isoleucine and leucine showed slightly increased cytotoxicity $(67-74 \%$ cell viability), whereas those freeze-dried with lysine exhibited strong cytotoxicity (11\% cell viability) (Fig. 4). In contrast, the
siRNA lipoplexes freeze-dried with the other amino acids did not induce a significant cytotoxic effect.

Characterization of freeze-dried siRNA lipoplexes after reconstitution. To examine whether the amino acids used during freeze-drying affected the size of siRNA lipoplexes after rehydration, freeze-dried siRNA lipoplexes in the presence of $100 \mathrm{mM}$ amino acid solutions were prepared, and the sizes of the resulting siRNA lipoplexes after rehydration were measured. The cationic liposomes were $\sim 100 \mathrm{~nm}$ in size, and the siRNA lipoplexes were $\sim 180 \mathrm{~nm}$ (data not shown) before freeze-drying. After freeze-drying and rehydration, the sizes of the siRNA lipoplexes ranged from 190-2,900 nm, although the lipoplexes freeze-dried with arginine, histidine, leucine and lysine were aggregated (Table I). The siRNA lipoplexes freeze-dried with cysteine, glycine, isoleucine, methionine or valine had larger sizes (800-2,900 nm). In contrast, siRNA lipoplexes freeze-dried with alanine, asparagine, glutamine, proline, serine or threonine were relatively smaller $(\sim 340$, $\sim 370, \sim 360, \sim 540, \sim 420$ and $\sim 190 \mathrm{~nm}$, respectively) with a monodisperse distribution (0.17-0.24 in PDI). Amongst these, polar but uncharged hydrophilic amino acids (asparagine, glutamine, serine and threonine) appeared effective for production of stable siRNA lipoplexes in size after freeze-drying (200-400 nm), indicating that amino acids that possessed an $-\mathrm{OH}$ or $-\mathrm{CONH}_{2}$ moiety in the side chain may interact with the polar head groups on the surface of the siRNA lipoplexes, and prevent the aggregation of siRNA lipoplexes caused by freeze-drying. From these results, the types of amino acids used during freeze-drying affected the size of the siRNA lipoplexes after rehydration.

\section{Discussion}

In Rev-transfection, freeze-drying of lipoplexes containing Luc siRNA in the presence of $50-100 \mathrm{mM}$ amino acids strongly suppressed luciferase activity regardless of the amino acid type. However, the presence of alanine, glutamine, glycine, serine, threonine or valine in freeze-drying increased 


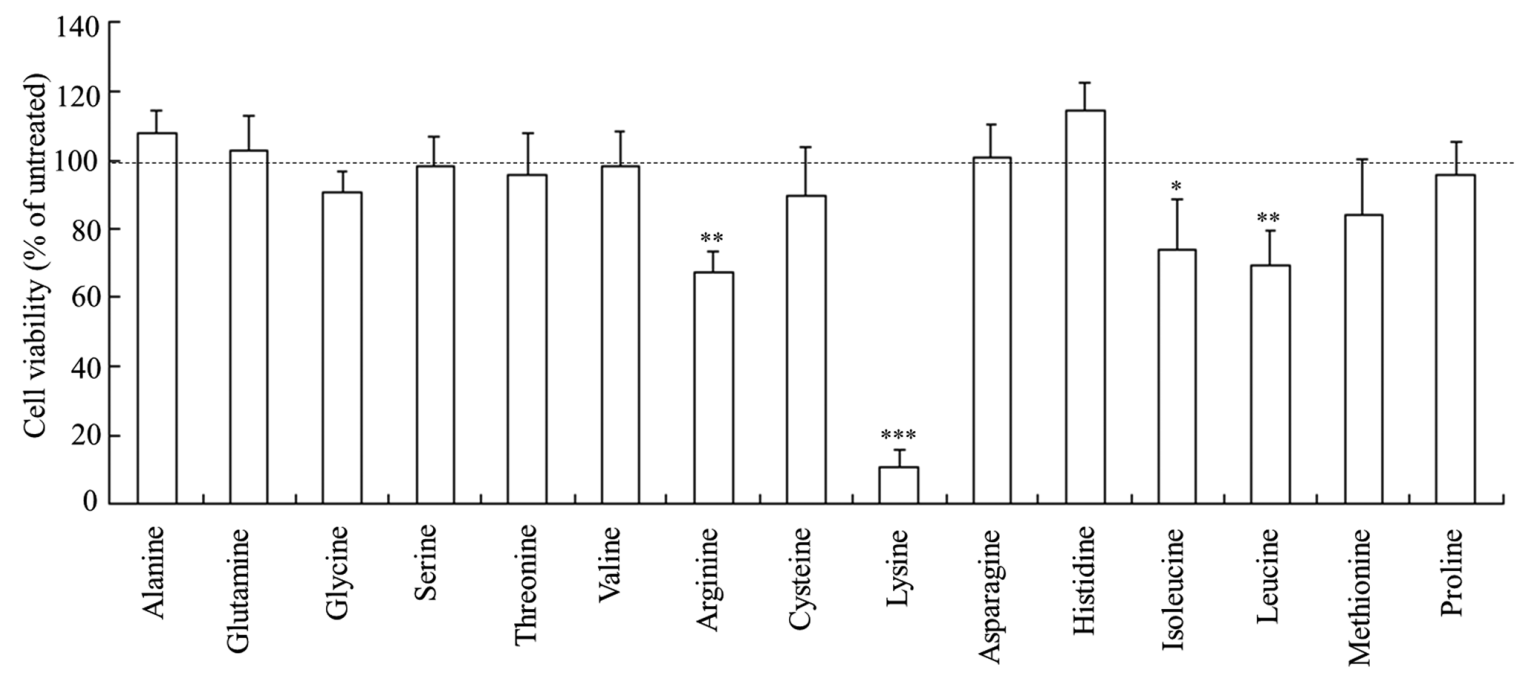

Figure 4. Cell viability $24 \mathrm{~h}$ after reverse transfection with freeze-dried siRNA lipoplexes. Lipoplexes containing 5 pmol Cont siRNA were diluted in a $12.5 \mu 1$ solution containing $100 \mathrm{mM}$ amino acids and then transferred to 96-well plates, followed by freeze-drying. MCF-7-Luc cells suspended in culture medium $(100 \mu \mathrm{l})$ were added to the plate (final siRNA concentration, $50 \mathrm{nM}$ ). Data are presented as the mean \pm standard deviation of at least three repeats. "P $<0.05$, ${ }^{* *} \mathrm{P}<0.01,{ }^{* * *} \mathrm{P}<0.001$ vs. untreated cells. Luc, luciferase; Cont, control; siRNA, small interfering RNA; lipoplex, liposome complex.

Table I. Particle size of small interfering RNA lipoplexes after rehydration of freeze-dried lipoplexes.

\begin{tabular}{lcc}
\hline $\begin{array}{l}\text { Amino acid used } \\
\text { for freeze-drying }\end{array}$ & $\begin{array}{c}\text { Size }^{\mathrm{a}, \mathrm{c}}, \\
\mathrm{nm}\end{array}$ & $\begin{array}{c}\text { Polydispersity } \\
\text { index }^{\mathrm{c}}\end{array}$ \\
\hline Alanine & $344.1 \pm 90.0$ & $0.18 \pm 0.02$ \\
Arginine & Aggregation & N.D. \\
Asparagine & $366.3 \pm 39.2$ & $0.20 \pm 0.02$ \\
Cysteine & $840.6 \pm 15.6$ & $0.32 \pm 0.01$ \\
Glutamine & $361.5 \pm 35.4$ & $0.17 \pm 0.01$ \\
Glycine & $2,443.6 \pm 178.6$ & $0.73 \pm 0.06$ \\
Histidine & Aggregation & N.D. \\
Isoleucine & $1,153.0 \pm 8.7$ & $0.36 \pm 0.01$ \\
Leucine & Aggregation & N.D. \\
Lysine & Aggregation & N.D. \\
Methionine & $2,916.5 \pm 100.2$ & $0.65 \pm 0.01$ \\
Proline & $540.6 \pm 42.3$ & $0.24 \pm 0.02$ \\
Serine & $420.8 \pm 12.6$ & $0.20 \pm 0.01$ \\
Threonine & $192.6 \pm 4.5$ & $0.24 \pm 0.02$ \\
Valine & $2,045.5 \pm 565.1$ & $0.60 \pm 0.16$ \\
\hline
\end{tabular}

${ }^{\mathrm{a}} 100 \mathrm{mM}$ amino acid solution; ${ }^{\text {bin }}$ water; ${ }^{\mathrm{c}}$ data are presented as the mean \pm standard deviation of three repeats. N.D., not determined.

luciferase activity in a concentration-dependent manner upon Rev-transfection with lipoplexes of Cont siRNA. It has been reported that genes were specifically upregulated in response to supra-physiological concentrations of amino acids (22). Increasing the concentration of amino acids from physiological to supraphysiological levels stimulates protein synthesis (23). Therefore, it was speculated that the increase in amino acid levels may activate the transcription and/or translation of certain genes in cells, resulting in an increase in luciferase activity. In contrast, a decrease in luciferase activity in the cells was observed after Rev-transfection with lipoplexes of Cont siRNA freeze-dried with arginine, cysteine or lysine. However, it was not clear why they exhibited decreased luciferase activity. Arginine and lysine are basic amino acids, and the presence of positively charged amino acids may lead to the downregulation of luciferase activity via cytotoxicity. With cysteine, it was speculated that the thiol groups of cysteine residues may affect luciferase activity, as cysteine is a potent nucleophile and is often linked to another cysteine to form a covalent disulfide bond. Furthermore, the presence of asparagine, histidine, isoleucine, leucine, methionine or proline in freeze-drying did not significantly affect the luciferase activity after Rev-transfection with lipoplexes of Cont siRNA, indicating that these amino acids may be suitable for freeze-drying siRNA lipoplexes. Amongst the amino acids tested in the present study, asparagine was suitable for Rev-transfection since siRNA lipoplexes freeze-dried with asparagine at concentrations $>100 \mathrm{mM}$ exhibited specific gene suppression, produced large cakes after freeze-drying and remained relatively small in size after rehydration (Table I). However, it is not clear why asparagine is an effective cryoprotectant for the freeze-drying of siRNA lipoplexes. In our previous study, it was shown that Rev-transfection with siRNA lipoplexes freeze-dried in a $25 \mathrm{mM}$ disaccharide or trisaccharide solution could induce efficient gene knockdown, and that the presence of $100 \mathrm{mM}$ saccharide during freeze-drying did not substantially increase the size of the siRNA lipoplexes $(\sim 200 \mathrm{~nm})(7,8)$. Based on these findings, saccharides might be more suitable as cryoprotectants for siRNA lipoplexes than amino acids. Forney-Stevens et al (24) demonstrated that 15 amino acids can significantly improve the storage stability of sucrose-based protein formulations after freeze-drying. Therefore, a combination of saccharides and amino acids may be an effective cryoprotectant for the freeze-drying of siRNA lipoplexes. However, further studies are required to investigate the optimal combination of saccharides and amino acids as a cryoprotectant for Rev-transfection.

In conclusion, in the present study, the effect of amino acids in the freeze-drying of siRNA lipoplexes on gene knockdown 
by Rev-transfection was assessed, and the results showed that the types of amino acids affected gene knockdown activity and lipoplex size after rehydration. Amongst the amino acids tested, the presence of asparagine in the freeze-drying of siRNA lipoplexes showed strong gene knockdown activity without a notable cytotoxic effect and had satisfactory particle sizes after rehydration. These findings provide valuable information regarding amino acids as cryoprotectants for Rev-transfection using freeze-dried siRNA lipoplexes for the efficient delivery of siRNA into cells.

\section{Acknowledgements}

We would like to thank Mr Ayato Kubota, Ms Natsuki Inagaki and Mr Shun Fujishita (Department of Molecular Pharmaceutics, Hoshi University) for assistance with the experimental work (in vitro gene-knockdown effect).

\section{Funding}

No funding was received.

\section{Availability of data and materials}

The datasets used and/or analyzed during the present study are available from the corresponding author on reasonable request.

\section{Authors' contributions}

YH conceived and designed the study. Experiments were performed by MT. YH and MT wrote the manuscript. Both authors have read and approved the final manuscript. MT and YH confirm the authenticity of all the raw data

\section{Ethics approval and consent to participate}

Not applicable.

\section{Patient consent for publication}

Not applicable.

\section{Competing interests}

The authors declare that they have no competing interests.

\section{References}

1. Wilson RC and Doudna JA: Molecular mechanisms of RNA interference. Annu Rev Biophys 42: 217-239, 2013.

2. Zhang MM, Bahal R, Rasmussen TP, Manautou JE and Zhong XB: The growth of siRNA-based therapeutics: Updated clinical studies. Biochem Pharmacol 189: 114432, 2021.

3. Thapa B, Remant KC and Uludağ H: siRNA library screening to identify complementary therapeutic pairs in triple-negative breast cancer cells. Methods Mol Biol 1974: 1-19, 2019.
4. Erfle H, Neumann B, Liebel U, Rogers P, Held M, Walter T, Ellenberg J and Pepperkok R: Reverse transfection on cell arrays for high content screening microscopy. Nat Protoc 2: 392-399, 2007.

5. Zhang S, Zhi D and Huang L: Lipid-based vectors for siRNA delivery. J Drug Target 20: 724-735, 2012.

6. Yadava P, Gibbs M, Castro C and Hughes JA: Effect of lyophilization and freeze-thawing on the stability of siRNA-liposome complexes. AAPS PharmSciTech 9: 335-341, 2008.

7. Hattori Y, Hu S and Onishi H: Effects of cationic lipids in cationic liposomes and disaccharides in the freeze-drying of siRNA lipoplexes on gene silencing in cells by reverse transfection. J Liposome Res 30: 235-245, 2020.

8. Tang M, Hu S and Hattori Y: Effect of pre freezing and saccharide types in freeze drying of siRNA lipoplexes on gene silencing effects in the cells by reverse transfection. Mol Med Rep 22: 3233-3244, 2020.

9. Franzé S, Selmin F, Samaritani E, Minghetti P and Cilurzo F: Lyophilization of liposomal formulations: Still necessary, still challenging. Pharmaceutics 10: 10, 2018.

10. Abdelwahed W, Degobert G, Stainmesse S and Fessi H: Freezedrying of nanoparticles: Formulation, process and storage considerations. Adv Drug Deliv Rev 58: 1688-1713, 2006.

11. Ball RL, Bajaj P and Whitehead KA: Achieving long-term stability of lipid nanoparticles: Examining the effect of $\mathrm{pH}$, temperature, and lyophilization. Int J Nanomedicine 12: 305-315, 2016.

12. Kundu AK, Chandra PK, Hazari S, Ledet G, Pramar YV, Dash S and Mandal TK: Stability of lyophilized siRNA nanosome formulations. Int J Pharm 423: 525-534, 2012.

13. Andersen MØ, Howard KA, Paludan SR, Besenbacher F and Kjems J: Delivery of siRNA from lyophilized polymeric surfaces. Biomaterials 29: 506-512, 2008.

14. Mohammed AR, Coombes AG and Perrie Y: Amino acids as cryoprotectants for liposomal delivery systems. Eur J Pharm Sci 30: 406-413, 2007.

15. Crowe JH and Crowe LM: Factors affecting the stability of dry liposomes. Biochim Biophys Acta 939: 327-334, 1988.

16. Koster KL, Webb MS, Bryant G and Lynch DV: Interactions between soluble sugars and POPC (1-palmitoyl-2-oleoylphosphatidylcholine) during dehydration: Vitrification of sugars alters the phase behavior of the phospholipid. Biochim Biophys Acta 1193: 143-150, 1994.

17. Stärtzel P: Arginine as an excipient for protein freeze-drying: A mini review. J Pharm Sci 107: 960-967, 2018.

18. Paik SH, Kim YJ, Han SK, Kim JM, Huh JW and Park YI: Mixture of three amino acids as stabilizers replacing albumin in lyophilization of new third generation recombinant factor VIII GreenGene F. Biotechnol Prog 28: 1517-1525, 2012.

19. Hattori Y, Nakamura T, Ohno H, Fujii N and Maitani Y: siRNA delivery into tumor cells by lipid-based nanoparticles composed of hydroxyethylated cholesteryl triamine. Int J Pharm 443: 221-229, 2013.

20. Hattori Y, Hara E, Shingu Y, Minamiguchi D, Nakamura A, Arai S, Ohno H, Kawano K, Fujii N and Yonemochi E: siRNA delivery into tumor cells by cationic cholesterol derivative-based nanoparticles and liposomes. Biol Pharm Bull 38: 30-38, 2015.

21. Hattori Y, Nakamura M, Takeuchi N, Tamaki K, Ozaki K and Onishi H: Effect of cationic lipid type in pegylated liposomes on siRNA delivery following the intravenous injection of siRNA lipoplexes. World Acad Sci 1: 74-85, 2019.

22. Varga J, Li L, Mauviel A, Jeffrey J and Jimenez SA: L-Tryptophan in supraphysiologic concentrations stimulates collagenase gene expression in human skin fibroblasts. Lab Invest 70: 183-191, 1994.

23. Vary TC, Jefferson LS and Kimball SR: Amino acid-induced stimulation of translation initiation in rat skeletal muscle. Am J Physiol 277: E1077-E1086, 1999.

24. Forney-Stevens KM, Bogner RH and Pikal MJ: Addition of amino acids to further stabilize lyophilized sucrose-based protein formulations: I. Screening of 15 amino acids in two model proteins. J Pharm Sci 105: 697-704, 2016. 